AperTO - Archivio Istituzionale Open Access dell'Università di Torino

\title{
Cohen operators associated with signal representations
}

\section{This is the author's manuscript}

Original Citation:

\section{Availability:}

This version is available http://hdl.handle.net/2318/1652801 since 2018-01-09T13:20:36Z

Publisher:

Ivan E. Egorov, Sergey V. Popov, Petr N. Vabishchevich, Mikhail Yu. Antonov, Nyurgun P. Lazarev,

Published version:

DOI:10.1063/1.5012677

Terms of use:

Open Access

Anyone can freely access the full text of works made available as "Open Access". Works made available under a Creative Commons license can be used according to the terms and conditions of said license. Use of all other works requires consent of the right holder (author or publisher) if not exempted from copyright protection by the applicable law. 


\title{
Cohen Operators Associated with Signal Representations
}

\author{
Paolo Boggiatto ${ }^{1, b)}$, Evanthia Carypis ${ }^{2, c)}$ and Alessandro Oliaro ${ }^{3, a)}$ \\ ${ }^{1}$ Department of Mathematics, University of Torino, Via Carlo Alberto, 10, I-10123 Torino (TO), Italy \\ ${ }^{2}$ Department of Mathematics, University of Torino, Via Carlo Alberto, 10, I-10123 Torino (TO), Italy \\ ${ }^{3}$ Department of Mathematics, University of Torino, Via Carlo Alberto, 10, I-10123 Torino (TO), Italy

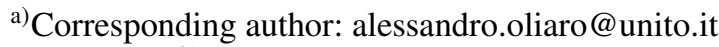 \\ b) paolo.boggiatto@unito.it \\ c) evanthia.carypis@unito.it
}

\begin{abstract}
In this paper we study the relationships between general quantization rules which yield Cohen operators and the signal time-frequency representations in the Cohen class. After defining a suitable functional setting, we focus on the particular case of the concentration operators which, as the multipliers and Fourier multipliers in the Donoho-Stark uncertainty principle, furnish estimates of the energy concentration on subsets of the time-frequency space. Finally we study the case of positive representations in the Cohen class.
\end{abstract}

\section{INTRODUCTION}

Time-frequency analysis of signals is an important area of research where techniques of harmonic analysis are widely used. Classically the time representation of a signal is a complex function (or distribution) $f(x)$ with $x \in \mathbb{R}$ (or for more of generality $x \in \mathbb{R}^{d}$ ), whereas its frequency representation is the Fourier transform

$$
\widehat{f}(\omega)=\int_{\mathbb{R}^{d}} f(x) e^{-2 \pi i \omega x} d x .
$$

The quantities $|f(x)|^{2}$ and $|\widehat{f}(\omega)|^{2}$ are the corresponding distributions of energy in the case $f \in L^{2}\left(\mathbb{R}^{d}\right)$. However since the mid 1900s a vast literature has been developed in order to find convenient representations of signals simultaneously with respect to time and frequency. The Wigner trasform

$$
f(x) \longrightarrow \operatorname{Wig}(f)(x, \omega)=\int_{\mathbb{R}^{d}} e^{-2 \pi i \omega t} f(x+t / 2) \overline{f(x-t / 2)} d t,
$$

defined by E. Wigner in the context of quantum physics, is of fundamental importance also as example of timefrequency representation (see [5], [8], [15]). Based on this transform the Cohen class of time-frequency representations is defined as the set of quadratic forms of the type

$$
f(x) \longrightarrow Q_{\sigma}(f)(x, \omega)=(\sigma * \operatorname{Wig}(f))(x, \omega),
$$

where $\sigma(x, \omega)$ is the so-colled Cohen kernel (note that $\operatorname{Wig}(f)$ is obtained for $\sigma=\delta$, the Dirac delta). Clearly $f(x)$ and $\sigma(x, \omega)$ must be chosen in functional (or distributional) spaces such that the convolution in the time-frequency space $\mathbb{R}_{x}^{d} \times \mathbb{R}_{\omega}^{d}$ appearing in (2) makes sense. To each quadratic form there corresponds a sesquilinear form $Q_{\sigma}(f, g)(x, \omega)$ defined in obvious way. The remarkable unifying approach of the Cohen class resides in the fact that essentially all of the most used quadratic forms can be obtained by suitable choices of the Cohen kernel.

Any time-frequency representation $Q_{\sigma}$ defines an operator $T_{\sigma}^{a}$, depending on a symbol $a(x, \omega)$, by the formula (see [5], [6]):

$$
\left(T_{\sigma}^{a} f, g\right)=\left(a, Q_{\sigma}(g, f)\right)
$$


where the brackets are intended in $L^{2}$, or more generally in distributional sense (we consider distributions as conjugate linear forms). The operators $T_{\sigma}^{a}$ obtained in this way are called Cohen operators. Actually, under some technical hypotheses, (3) establishes a bijection between sesquiliear forms $Q_{\sigma}$ and quantizations rules $a \rightarrow T_{\sigma}^{a}$, connecting therefore the study of time-frequency representations with that of Cohen operators (see section "Cohen operators"). As for representations, essentially all types of commonly used pseudo-differential operators (Weyl, Kohn-Nirenberg, Localization operators, etc.) can be obtained with particular choices of the Cohen kernel $\sigma(x, \omega)$ (see e.g. [5], [19], [20], [21], [22]).

In this paper we study the behavior of Cohen operators with respect to boundedness, adjoints and composition (see section "Cohen operators"). We consider then (in the section "Concentration operators") the case of concentration operators which consists of Cohen operators with characteristic functions as symbols and show how these generalize the multipliers and Fourier multipliers operators which are used in the Donoho-Stark uncertainty principle [12]. This enlarges the useful tools for expressing concentration of a signal on sets in the time-frequency space and we give some applications of this fact in terms of uncertainty principles involving concentration operators (see section "Positive forms and uncertainty principle"). Among the wide literature about uncertainty principles in time-frequency analysis see e.g. [1], [2], [3], [4], [7], [9], [13], [16], [18]. The last section is motivated by the remark that the lack of positivity of the representations $Q_{\sigma}$ constitutes one of the main differences between the concept of "energy distributions" in signal theory (or quantum mechanincs) and that of "probability, or mass, distributions" in statistics and classical physics ([8], [10], [11]). It is therefore of interest to investigate cases when $Q_{\sigma}$ is positive, as these cases can be considered, in some sense, the closest to the classical ones. We present some results along these lines in the section "Positive forms and uncertainty principle" and conclude presenting the above-mentioned form of uncertainty principle.

\section{COHEN OPERATORS}

In a general sense we call quantization (or quantization rule) any linear map associating a symbol $a(x, \omega)$ belonging to a Banach (or Fréchet) space of functions or distributions with a linear operator acting between Banach (or Fréchet) spaces.

We start this section by considering a bijective correspondence between vector valued sesquilinear forms and quatizations (see [6]).

Proposition 1 Let $E, E_{1}, E_{2}$ be Banach spaces, and $E_{2}$ be reflexive. Then

(i) If $\varphi: E_{2} \times E_{1} \rightarrow E$ is a bounded sesquilinear form then there exists a unique bounded linear map a $\in E^{*} \rightarrow$ $T_{a} \in B\left(E_{1}, E_{2}^{*}\right)$ such that

$$
\left(T^{a} u, v\right)=(a, \varphi(v, u)) \quad\left(\text { for } u \in E_{1}, v \in E_{2}\right) .
$$

(ii) If $a \in E^{*} \rightarrow T_{a} \in B\left(E_{1}, E_{2}^{*}\right)$ is a bounded linear map, then (4) defines a unique bounded sesquilinear map $\varphi: E_{2} \times E_{1} \rightarrow E$.

As particular case of this general result we have a bijective correspondence between Cohen class time-frequency representations and Cohen operators. This can be made precise in various functional and distributional settings, we present some of them in the following properties. We start by tempered distributions and Schwarz functions.

Corollary $1 \quad$ Let $\sigma \in \mathcal{S}\left(\mathbb{R}^{2 d}\right)$ be a fixed Cohen kernel. Then the definition of a quantization rule a $\longrightarrow T_{\sigma}^{a}$ is equivalent to the definition of a time-frequency representation $Q_{\sigma}(f)=\sigma *$ Wig $(f)$, the two being related by the formula

$$
\left(T_{\sigma}^{a} f, g\right)=\left(a, Q_{\sigma}(g, f)\right), \quad \text { with } a \in \mathcal{S}\left(\mathbb{R}^{2 d}\right), f, g \in \mathcal{S}\left(\mathbb{R}^{d}\right) .
$$

As the $L^{2}$ norm is physically interpreted as the energy of a signal, one of the most natural setting for identity (5) is clearly the case $f, g \in L^{2}\left(\mathbb{R}^{d}\right)$. We state therefore the following boundedness result in this setting.

Proposition 2 Let $f, g \in L^{2}\left(\mathbb{R}^{d}\right), a \in L^{p}\left(\mathbb{R}^{2 d}\right)$ and $\sigma \in L^{q}\left(\mathbb{R}^{2 d}\right)$ with $\frac{1}{p}+\frac{1}{q}=\frac{3}{2}$; then the operator $T_{\sigma}^{a}$ defined by (5) is bounded on $L^{2}\left(\mathbb{R}^{d}\right)$ with norm estimate

$$
\left\|T_{\sigma}^{a}\right\|_{B\left(L^{2}\left(\mathbb{R}^{d}\right)\right)} \leq\|a\|_{L^{p}\left(\mathbb{R}^{2 d}\right)}\|\sigma\|_{L^{q}\left(\mathbb{R}^{2 d}\right)} .
$$

Furthermore in this case we also have a bounded sesquilinear form $Q_{\sigma}: L^{2}\left(\mathbb{R}^{d}\right) \times L^{2}\left(\mathbb{R}^{d}\right) \longrightarrow L^{p^{\prime}}\left(\mathbb{R}^{2 d}\right)$, with $\frac{1}{p}+\frac{1}{p^{\prime}}=1$ with same norm estimate. 
Proof. Setting $\tilde{\sigma}(x, \omega)=\sigma(-x,-\omega)$ and recalling that $\|\operatorname{Wig}(g, f)\|_{L^{2}\left(\mathbb{R}^{2 d}\right)}=\|g\|_{L^{2}\left(\mathbb{R}^{d}\right)}\|f\|_{L^{2}\left(\mathbb{R}^{d}\right)}$ we have

$$
\begin{aligned}
\left|\left(T_{\sigma}^{a} f, g\right)\right| & =|(a, \sigma * \operatorname{Wig}(g, f))| \\
& =|(a * \overline{\widetilde{\sigma}}, \operatorname{Wig}(g, f))| \\
& \leq\|a * \overline{\widetilde{\sigma}}\|_{2}\|\operatorname{Wig}(g, f)\|_{2} \\
& \leq\|a\|_{p}\|\sigma\|_{q}\|f\|_{2}\|g\|_{2} .
\end{aligned}
$$

This proves the boundedness of the operator $T_{\sigma}^{a}$. The remaining statement about the boundedness of $Q_{\sigma}$ is a straightforward consequence of (4).

As mentioned in the introduction, all most used quadratic time-frequency representations belong to the Cohen class. Unfortunately in several important cases the Cohen kernel does not belong to any $L^{p}\left(\mathbb{R}^{2 d}\right)$ space with $p<\infty$ and therefore, as can easily be checked, the previous result can not be applied. The following proposition shows that if however the Fourier transform of the Cohen kernel belongs to $L^{\infty}$ we still have interesting cases of boundedness. This is for example the case of the well-known generalizations of the Wigner transform called $\tau$-Wigner, for $\tau \in[0,1]$, which, for $\tau \neq \frac{1}{2}$, can be defined by the Cohen kernels $\sigma_{\tau}=\frac{2^{d}}{|2 \tau-1|^{d}} e^{2 \pi i \frac{2}{2 \tau-1} x \omega}$, (see [6]).

Proposition 3 Let $f, g \in L^{2}\left(\mathbb{R}^{d}\right), a \in L^{2}\left(\mathbb{R}^{2 d}\right)$ and $\widehat{\sigma} \in L^{\infty}\left(\mathbb{R}^{2 d}\right)$; then (5) defines a bounded operator $T_{\sigma}^{a}$ on $L^{2}\left(\mathbb{R}^{d}\right)$ and

$$
\left\|T_{\sigma}^{a}\right\|_{B\left(L^{2}\left(\mathbb{R}^{d}\right)\right)} \leq\|a\|_{L^{2}\left(\mathbb{R}^{2 d}\right)}\|\widehat{\sigma}\|_{L^{\infty}\left(\mathbb{R}^{2 d}\right)} .
$$

Proof. By the Plancherel theorem and again the equality $\|\operatorname{Wig}(g, f)\|_{L^{2}\left(\mathbb{R}^{2 d}\right)}=\|g\|_{L^{2}\left(\mathbb{R}^{d}\right)}\|f\|_{L^{2}\left(\mathbb{R}^{d}\right)}$, we have

$$
\begin{aligned}
\left|\left(T_{\sigma}^{a} f, g\right)\right| & =|(a, \sigma * \operatorname{Wig}(g, f))| \\
& =\mid \widehat{a}, \widehat{\sigma} \widehat{\operatorname{Wig}}(g, f)) \mid \\
& \leq\|a\|_{L^{2}\left(\mathbb{R}^{2 d}\right)}\|\widehat{\sigma}\|_{L^{\infty}\left(\mathbb{R}^{2 d}\right)}\|g\|_{2}\|f\|_{2} .
\end{aligned}
$$

The following is a boundedness result under a slightly different point of view. It states that if a representation is well-defined on all signals then the corresponding Cohen operator is automatically bounded.

Proposition $4 \quad$ Suppose that $a \in L^{p}\left(\mathbb{R}^{2 d}\right), \frac{1}{p}+\frac{1}{p^{\prime}}=1$, and $Q_{\sigma}: L^{2}\left(\mathbb{R}^{d}\right) \times L^{2}\left(\mathbb{R}^{d}\right) \longrightarrow L^{p^{\prime}}\left(\mathbb{R}^{2 d}\right)$ is well-defined. Then the operator $T_{\sigma}^{a}$ defined by (5) is $L^{2}$-bounded (and consequently also the form $Q_{\sigma}: L^{2} \times L^{2} \longrightarrow L^{p^{\prime}}$ ).

Proof. By the hypothesis and (5) we have that $\left(T_{\sigma}^{a} f, g\right)$ is well-defined and finite for all $f, g \in L^{2}\left(\mathbb{R}^{d}\right)$, this implies that $T_{\sigma}^{a}$ is a linear operator everywhere defined on $L^{2}\left(\mathbb{R}^{d}\right)$ with values in $L^{2}\left(\mathbb{R}^{d}\right)$. The (a priori unbounded) adjoint operator therefore also acts from $L^{2}\left(\mathbb{R}^{d}\right)$ into itself. We apply now the closed graph Theorem. Suppose $f_{n} \rightarrow f$ and $T_{\sigma}^{a} f_{n} \rightarrow g$ in $L^{2}\left(\mathbb{R}^{d}\right)$; then for every $h \in L^{2}\left(\mathbb{R}^{d}\right)$

$$
(g, h)=\lim _{n \rightarrow \infty}\left(T_{\sigma}^{a} f_{n}, h\right)=\lim _{n \rightarrow \infty}\left(f_{n},\left(T_{\sigma}^{a}\right)^{*} h\right)=\left(f,\left(T_{\sigma}^{a}\right)^{*} h\right)=\left(T_{\sigma}^{a} f, h\right)
$$

which means $T_{\sigma}^{a} f=g$.

We study next some basic properties of the Cohen operators (adjoints, etc). For simplicity we state the results in the case of Schwarz functions, they could however be reformulated in larger functional settings with minor modifications. We recall that the classical Weyl operators are Cohen operators associated with Cohen kernel $\sigma=\delta$, i.e. associated with the Wigner representation, in symbols:

$$
\left(W^{a} f, g\right)=(a, \operatorname{Wig}(g, f)),
$$

where $W^{a}$ denotes the Weyl operator with symbol $a$.

Proposition 5 Let $f, g \in \mathcal{S}\left(\mathbb{R}^{d}\right), \sigma \in \mathcal{S}\left(\mathbb{R}^{2 d}\right), a \in \mathcal{S}\left(\mathbb{R}^{2 d}\right)$ as before. Then

1) $T_{\sigma}^{a}=W^{b}$ with $b=a * \widetilde{\bar{\sigma}}$;

2) $T_{\sigma}^{a}=T_{\overline{\bar{a}}}^{\widetilde{\widetilde{\sigma}}}$; 
3) $T_{\sigma}^{1}=c$ Id where $c=\int_{\mathbb{R}^{2 d}} \overline{\sigma(x, \omega)} d x d y$;

4) $\left(T_{\sigma}^{a}\right)^{*}=T_{\bar{\sigma}}^{\bar{a}}$;

5) For $\sigma_{j} \in \mathcal{S}\left(\mathbb{R}^{2 d}\right), a_{j} \in \mathcal{S}\left(\mathbb{R}^{2 d}\right), j=1,2$, the operator $T_{a_{2}}^{\sigma_{2}} \circ T_{a_{1}}^{\sigma_{1}}$ has Schwartz kernel:

$$
k(y, t)=\int \mathcal{F}_{2}^{-1}\left[a_{2} * \overline{\widetilde{\sigma_{2}}}\right]\left(\frac{y+x}{2}, y-x\right) \mathcal{F}_{2}^{-1}\left[a_{1} * \overline{\widetilde{\sigma_{1}}}\right]\left(\frac{x+t}{2}, x-t\right) d x .
$$

Proof. Points 1) to 3) are immediate consequences of the definitions and elementary properties of convolutions. Point 4) follows from equation (5) and the fact that

$$
\overline{Q_{\sigma}(f, g)}=Q_{\bar{\sigma}}(g, f)
$$

Concerning point 5) we have

$$
\begin{aligned}
\left(T_{a_{2}}^{\sigma_{2}} \circ T_{a_{1}}^{\sigma_{1}} f, g\right) & =\left(a_{2} * \overline{\widetilde{\sigma_{2}}}, \operatorname{Wig}\left(g, T_{\sigma_{1}}^{a_{1}} f\right)\right) \\
& =\int\left(a_{2} * \overline{\widetilde{\sigma_{2}}}\right)(x, \omega) e^{2 \pi i \omega t} T_{\sigma_{1}}^{a_{1}} f\left(x-\frac{t}{2}\right) \overline{g\left(x+\frac{t}{2}\right)} d t d x d \omega \\
& =\int\left(a_{2} * \overline{\widetilde{\sigma_{2}}}\right)\left(\frac{x+t}{2}, \omega\right) e^{2 \pi i \omega(x-t)} T_{\sigma_{1}}^{a_{1}} f(t) \overline{g(x)} d t d x d \omega \\
& =\int\left(a_{2} * \overline{\widetilde{\sigma_{2}}}\right)\left(\frac{x+t}{2}, \omega\right) e^{2 \pi i \omega(x-t)} e^{2 \pi i \omega^{\prime}\left(t-t^{\prime}\right)}\left(a_{1} * \overline{\sigma_{1}}\right)\left(\frac{t+t^{\prime}}{2}, \omega^{\prime}\right) f\left(t^{\prime}\right) \overline{g(x)} d t^{\prime} d \omega^{\prime} d t d x d \omega \\
& =\int \mathcal{F}_{\omega \rightarrow(x-t)}^{-1}\left[\left(a_{2} * \overline{\widetilde{\sigma_{2}}}\right)\left(\frac{x+t}{2}, \omega\right)\right]_{\mathcal{F}^{\prime} \rightarrow\left(t-t^{\prime}\right)}^{-1}\left[\left(a_{1} * \overline{\widetilde{\sigma_{1}}}\right)\left(\frac{t+t^{\prime}}{2}, \omega^{\prime}\right)\right] f\left(t^{\prime}\right) \overline{g(x)} d t^{\prime} d t d x .
\end{aligned}
$$

Hence the Schwarz kernel $k(x, y)$ of the composition $T_{a_{2}}^{\sigma_{2}} \circ T_{a_{1}}^{\sigma_{1}}$ is

$$
k(y, t)=\int \mathcal{F}_{2}^{-1}\left[a_{2} * \overline{\widetilde{\sigma_{2}}}\right]\left(\frac{y+x}{2}, y-x\right) \mathcal{F}_{2}^{-1}\left[a_{1} * \overline{\widetilde{\sigma_{1}}}\right]\left(\frac{x+t}{2}, x-t\right) d x .
$$

Remark 1 Part 4) of the previous proposition reveals a crucial fact about the relation between the general concept of quantization above introduced as intended in signal theory, and the more strict meaning of this term used in quantum mechanics where it is required that real symbols yield self-adjoint operators. This happens in our framework exactly when the Cohen kernel of the form associated with the quantization is real. For example it becomes clear that among all $\tau$-Weyl quantizations associated with $\tau$-Wigner forms (see above before Prop. 3 ), only the Weyl quantization is a "true" quantization in terms of physical meaning.

\section{CONCENTRATION OPERATORS}

In applications it is often of interest to know how much energy of a signal is concentrated on a fixed region of the time domain, of the frequency domain, or of the time-frequency plane. For a region $\Omega \subset \mathbb{R}_{x}^{d}$ located in the time domain the concentration of a signal $f$ is taken into account in a natural way by the cutoff operator $P_{\Omega} f=\chi_{\Omega}(x) f(x)$ (where $\chi_{\Omega}(x)=1$ for $x \in \Omega$ and zero otherwise). Analogously for a region $T \subset \mathbb{R}_{\omega}^{d}$ of the frequency domain it is natural to consider the Fourier multiplier operator $Q_{T} f=\mathcal{F}^{-1}\left[\chi_{T}(\omega) \widehat{f}(\omega)\right]$. In order to estimate the energy concentration on a subset $\Omega \times T$ of the time-frequency plane the composition $P_{\Omega} Q_{T}$ can be considered and this operator plays in fact a key role for example in the Donoho-Stark uncertainty principle (see [12]). In this section we show that $P_{\Omega} Q_{T}$ is a Cohen operator associated with the Rihaczek form (see below) and symbol $a(x, \omega)=\chi_{\Omega \times T}(x, \omega)$. It will therefore be natural to substitute the Rihaczek representations with general representations $Q_{\sigma}$ in the Cohen class and $\chi_{\Omega \times T}(x, \omega)$ with the characteristic function $\chi_{M}(x, \omega)$ of a general subset $M$ of the time-frequency plane, considering then as concentration operators the corresponding Cohen operators $T_{\sigma}^{\chi_{M}}$. 
Proposition 6 Let $f, g \in L^{2}\left(\mathbb{R}^{d}\right)$. We have

$$
\left(P_{\Omega} Q_{T} f, g\right)=\left(\chi_{\Omega \times T}, R(g, f)\right)
$$

where $R(g, f)=e^{-2 \pi i x \omega} g(x) \overline{\hat{f}(\omega)}$ is the Rihaczeck representation.

Proof. By the definition of $P_{\Omega}$ and $Q_{T}$ we have

$$
\begin{aligned}
\left(P_{\Omega} Q_{T} f, g\right) & =\left(\chi_{\Omega}(x) \int_{\mathbb{R}^{d}} e^{2 \pi i x \omega} \chi_{T}(\omega) \hat{f}(\omega) d \omega, g(x)\right) \\
& =\int_{\mathbb{R}^{2 d}} e^{2 \pi i x \omega} \chi_{\Omega}(x) \chi_{T}(\omega) \hat{f}(\omega) \overline{g(x)} d \omega d x \\
& =\left(\chi_{\Omega \times T}, R(g, f)\right) .
\end{aligned}
$$

Remark 2 Since the Rihaczeck representation is a particular representation in the Cohen class, namely,

$$
R(f, g)(x, \omega)=Q_{\sigma}(f, g)(x, \omega)
$$

for $\sigma(x, \omega)=2^{d} e^{-4 \pi i x \omega}$ (see for example [6]), we have that the composition $P_{\Omega} Q_{T}$ is the Cohen operator $T_{\sigma}^{a}$ (in the sense of (3)), with $a=\chi_{\Omega \times T}$ and $\sigma(x, \omega)=2^{d} e^{-4 \pi i x \omega}$.

Since the operator $P_{\Omega} Q_{T}$ can be considered as a way to measure the energy concentration of a signal in the region $\Omega \times T$ of the time-frequency plane, and this operator is connected with a specific representation in the Cohen class, we are led to the following definition of concentration operator associated to a time-frequency representation.

Definition $1 \quad$ Let $M \subset \mathbb{R}_{x}^{d} \times \mathbb{R}_{\omega}^{d}$ be a measurable set, $f, g \in \mathcal{S}\left(\mathbb{R}^{d}\right)$ and $\sigma \in \mathcal{S}\left(\mathbb{R}^{2 d}\right)$. Then the concentration operator associated with the set $M$ and the representation $Q_{\sigma}$ is the Cohen operator $T_{\sigma}^{\chi M}$. We denote such operator by $C_{\sigma}^{M}$, i.e. we have

$$
\left(C_{\sigma}^{M} f, g\right)=\left(\chi_{M}, Q_{\sigma}(g, f)\right)
$$

Remark 3 As usual we can consider more general functional settings in the definition of concentration operators, cf. for example Propositions 2, 3, 4, or the general correspondence given in Proposition 1.

We observe moreover that the concentration operator is a known object, see for example [17], where it is denoted by $L_{M}$ and is defined by the relation

$$
\left(L_{M} f, f\right)=\int_{M} Q_{\sigma}(f)(x, \omega) d x d \omega
$$

for $f \in \mathcal{S}\left(\mathbb{R}^{d}\right)$ and a Cohen representation $Q_{\sigma}$. Observe that in our case

$$
\left(C_{\sigma}^{M} f, f\right)=\left(\chi_{M}, Q_{\sigma}(f)\right)=\int_{M} \overline{Q_{\sigma}(f)(x, \omega)} d x d \omega,
$$

so $C_{\sigma}^{M}$ coincides with the operator $L_{M}$ associated to $\overline{Q_{\sigma}(f)}$.

Remark 4 From Proposition 5 we have that the adjoint of the concentration operator is given by

$$
\left(C_{\sigma}^{M}\right)^{*}=C_{\bar{\sigma}}^{M}
$$

then, since $\overline{Q_{\bar{\sigma}}(g, f)}=Q_{\sigma}(f, g)$, we have that

$$
\left(\left(C_{\sigma}^{M}\right)^{*} f, g\right)=\int_{M} Q_{\sigma}(f, g) d x d \omega
$$

In the particular case of the Rihaczeck representation, we have that $R(f, g)=\overline{R^{*}(g, f)}$, where $R^{*}(g, f)$ is the so-called “conjugate Rihaczeck” representation, defined as $R^{*}(g, f)=e^{2 \pi i x \omega} \overline{f(x)} \hat{g}(\omega)$. In the particular case when the set $M$ is of the form $\Omega \times T$ we have from Remark 2 that the corresponding concentration operator is $\left(P_{\Omega} Q_{T}\right)^{*}$, and since $P_{\Omega}$ and $Q_{T}$ are self-adjoint, we finally obtain that $Q_{T} P_{\Omega}$ is the concentration operator associated with the set $\Omega \times T$ and the conjugate Rihaczeck representation. 
We want now to find the kernel of the operator $C_{\sigma}^{M}$; we have the following result.

Proposition 7 Given $\sigma \in \mathcal{S}\left(\mathbb{R}^{2 d}\right)$ and a representation $Q_{\sigma}$ of the form (2), the corresponding concentration operator $C_{\sigma}^{M}$ satisfies

$$
C_{\sigma}^{M} f(x)=\int_{\mathbb{R}^{d}} k_{M}(x, y) f(y) d y
$$

for every $f \in \mathcal{S}\left(\mathbb{R}^{d}\right)$, where the kernel $k_{M}$ is given by

$$
k_{M}(x, y)=\mathcal{F}_{u \rightarrow \frac{x+y}{2}}\left[\check{\chi}_{M}(u, x-y) \widehat{\bar{\sigma}}(u, x-y)\right] .
$$

Proof. Let $T: \mathcal{S}\left(\mathbb{R}^{2 d}\right) \rightarrow \mathcal{S}\left(\mathbb{R}^{2 d}\right)$ be such that $T(F)(x, y)=F\left(x+\frac{y}{2}, x-\frac{y}{2}\right)$. Then we write the representation $Q_{\sigma}$ by using the following decomposition

$$
Q_{\sigma}(g, f)(x, \omega)=\mathcal{F}_{(u, t) \rightarrow(x, \omega)}\left\{\mathcal{F}_{\left(x^{\prime}, \omega^{\prime}\right) \rightarrow(u, t)}^{-1} \sigma\left(x^{\prime}, \omega^{\prime}\right) \mathcal{F}_{s \rightarrow u}^{-1}[T(g \otimes \bar{f})(s, t)]\right\} .
$$

Denoting by $\check{\chi}_{M}$ the inverse Fourier transform of $\chi_{M}$ we have

$$
\begin{aligned}
\iint_{M} \overline{Q_{\sigma}(g, f)(x, \omega)} d x d \omega & =\iint_{\mathbb{R}^{2 d}} \chi_{M}(x, \omega) \overline{\mathcal{F}_{(u, t) \rightarrow(x, \omega)}\left\{\mathcal{F}_{\left(x^{\prime}, \omega^{\prime}\right) \rightarrow(u, t)}^{-1} \sigma\left(x^{\prime}, \omega^{\prime}\right) \mathcal{F}_{s \rightarrow u}^{-1}[T(g \otimes \bar{f})(s, t)]\right\}} d x d \omega \\
& =\iint_{\mathbb{R}^{2 d}} \check{\chi}_{M}(u, t) \overline{\left\{\mathcal{F}_{\left(x^{\prime}, \omega^{\prime}\right) \rightarrow(u, t)}^{-1} \sigma\left(x^{\prime}, \omega^{\prime}\right) \mathcal{F}_{s \rightarrow u}^{-1}[T(g \otimes \bar{f})(s, t)]\right\}} d u d t,
\end{aligned}
$$

Applying Parseval formula with respect to the $u$-variable we then have

$$
\begin{aligned}
\iint_{M} Q_{\sigma} f(x, \omega) d x d \omega & =\iint_{\mathbb{R}^{2 d}}\left[\check{\chi}_{M}(u, t) \overline{\bar{\sigma}}(u, t)\right] \overline{\left[\mathcal{F}_{s \rightarrow u}^{-1}[T(g \otimes \bar{f})(s, t)]\right.} d u d t \\
& =\iint_{\mathbb{R}^{2 d}} \mathcal{F}_{u \rightarrow \xi}\left[\check{\chi}_{M}(u, t) \widehat{\bar{\sigma}}(u, t)\right] \overline{[T(g \otimes \bar{f})(\xi, t)]} d \xi d t \\
& =\iint_{\mathbb{R}^{2 d}} \mathcal{F}_{u \rightarrow \frac{x+y}{2}}\left[\check{\chi}_{M}(u, x-y) \widehat{\bar{\sigma}}(u, x-y)\right] f(y) \overline{g(x)} d x d y,
\end{aligned}
$$

and so from the definition of concentration operator it follows immediately that the integral kernel of $C_{\sigma}^{M}$ is given by (6).

\section{POSITIVE FORMS AND UNCERTAINTY PRINCIPLE}

In this section we focus on positive representations in the Cohen class. In time-frequency analysis the representations are not necessarily positive (for example, the Wigner itself is widely used, even though it is complex-valued); on the other hand, since a representation describes the energy distribution of a signal with respect to time and frequency, it is quite natural to study its positivity, or to look for positive representations. We focus in this section on a subclass of the Cohen's class, considering the representations of the form $\left|Q_{\sigma} f\right|$. We start by studying under which conditions $\left|Q_{\sigma}(f)\right|$ still belong to the Cohen's class; then we compute the trace class norm of the corresponding concentration operator $C_{\sigma}^{M}$, and prove a related uncertainty principle.

For $\alpha, \beta \in \mathbb{R}^{d}$ we denote by $T_{\alpha}$ and $M_{\beta}$ the translation and modulation operators, defined as

$$
T_{\alpha} f(x)=f(x-\alpha), \quad M_{\beta} f(x)=e^{2 \pi i \beta x} f(x),
$$

for a function (or distribution) $f$. We start by recalling the following property (cf. [14]).

Theorem 1 Suppose that a quadratic time-frequency representation $Q f$ is covariant and weakly continuous, that is, it satisfies

$$
Q\left(T_{\alpha} M_{\beta}\right)=T_{(\alpha, \beta)} Q f
$$

and

$$
|Q(f, g)(0,0)| \leqslant\|f\|_{2}\|g\|_{2}
$$


for all $f, g \in L^{2}\left(\mathbb{R}^{d}\right)$. Then there exists a tempered distribution $\sigma \in \mathcal{S}^{\prime}\left(\mathbb{R}^{2 d}\right)$ such that

$$
Q f=\sigma * \operatorname{Wig}(f),
$$

for all $f \in \mathcal{S}\left(\mathbb{R}^{d}\right)$.

We have the following result.

Proposition 8 Suppose $\sigma$ is real and belongs to $L^{p}\left(\mathbb{R}^{2 d}\right)$ with $p \in[1,2]$. The following statements hold for the Cohen class representation $Q_{\sigma}$ :

(i) $\left|Q_{\sigma}\right|$ belongs to the Cohen's class.

(ii) The concentration operator associated with $\left|Q_{\sigma}\right|$ is self-adjoint and positive.

Proof. (ii) is an easy consequence of the fact that $\left|Q_{\sigma}\right|(f, g)=\left|Q_{\sigma}\right|(g, f)$ and $\left|Q_{\sigma}\right|(f)>0$. In order to prove (i) it is enough, according to Theorem 1 , to shows that $\left|Q_{\sigma}\right|$ is covariant and weakly continuous. As $Q_{\sigma}$ is covariant, we have

$$
\left|Q_{\sigma}\left(T_{\alpha} M_{\beta} f\right)\right|=\left|T_{(\alpha, \beta)} Q_{\sigma}(f)\right|=T_{(\alpha, \beta)}\left|Q_{\sigma}(f)\right| .
$$

which proves that also $\left|Q_{\sigma}\right|$ is covariant. We show next the weak continuity. Without loss of generality, we can assume $\|\sigma\|_{p} \leqslant 2^{-d\left(p^{\prime}-2\right) / p^{\prime}}$. The Wigner transform is a bounded sesquilinear form on $L^{2}\left(\mathbb{R}^{d}\right) \times L^{2}\left(\mathbb{R}^{d}\right)$ with values both in $L^{2}\left(\mathbb{R}^{2 d}\right)$ and in $L^{\infty}\left(\mathbb{R}^{2 d}\right)$ and norm estimates

$$
\begin{aligned}
& \|\operatorname{Wig}(f, g)\|_{2} \leq\|f\|_{2}\|g\|_{2} \\
& \|\operatorname{Wig}(f, g)\|_{\infty} \leq 2^{d}\|f\|_{2}\|g\|_{2}
\end{aligned}
$$

An application of the Riesz-Thorin theorem for bilinear forms yields

$$
\|\operatorname{Wig}(f, g)\|_{p^{\prime}} \leq 2^{d\left(p^{\prime}-2\right) / p^{\prime}}\|f\|_{2}\|g\|_{2},
$$

and we have therefore

$$
\begin{aligned}
\left|Q_{\sigma}(f, g)(0,0)\right| & =\left|\int_{\mathbb{R}^{2 d}} \sigma(-s,-t) \operatorname{Wig}(f)(s, t) d s d t\right| \\
& \leqslant\|\sigma\|_{p}\|\operatorname{Wig}(f)\|_{p^{\prime}} \leq\|f\|_{2}\|g\|_{2}
\end{aligned}
$$

completing the proof that $\left|Q_{\sigma}\right|$ belongs to the Cohen's class.

We finally want to turn our attention to concentration operators as tools for showing that, in suitable sense, the subsets of the time-frequency space can not carry a "too large portion" of the energy of a signal. To this aim, we first recall, for completeness, the classical concept of $\varepsilon$-concentration of a signal and the Donoho-Stark uncertainty principle (see e.g. [14], Thm. 2.3.1) which is a well-known result in this direction.

We define then a concept of $\varepsilon$-concentration adapted to our context and dependent on a fixed concentration operator. We compute the trace-class norm of Cohen operators associated with positive representations and use this to obtain uncertainty principles which involve estimates of the measures of time-frequency space subsets in terms of our general concentration operators.

Definition 2 Given $\varepsilon \geq 0$, a function $f \in L^{2}\left(\mathbb{R}^{d}\right)$ is $\varepsilon$-concentrated on a measurable set $U \subseteq \mathbb{R}^{d}$ if

$$
\left(\int_{\mathbb{R}^{d} \backslash U}|f(x)|^{2} d x\right)^{1 / 2} \leq \varepsilon\|f\|_{2},
$$

or equivalently

$$
\left(\int_{U}|f(x)|^{2} d x\right)^{1 / 2} \geq \sqrt{1-\varepsilon^{2}}\|f\|_{2}
$$

Theorem 2 (Donoho-Stark) Suppose that $f \in L^{2}\left(\mathbb{R}^{d}\right), f \neq 0$, is $\varepsilon_{T}$-concentrated on $T \subseteq \mathbb{R}^{d}$, and $\widehat{f}$ is $\varepsilon_{\Omega^{-}}$ concentrated on $\Omega \subseteq \mathbb{R}^{d}$, with $T, \Omega$ measurable sets in $\mathbb{R}^{d}$ and $\varepsilon_{T}, \varepsilon_{\Omega} \geq 0$, $\varepsilon_{T}+\varepsilon_{\Omega} \leq 1$. Then

$$
|T||\Omega| \geq\left(1-\varepsilon_{T}-\varepsilon_{\Omega}\right)^{2} .
$$


Let now $M$ be a subset of the time-frequency space and $C_{\sigma}^{M}$ the concentration operator corresponding to $M$ and the positive representation $Q_{\sigma}$. Inspired by (9), we define $\varepsilon$-concentration with respect to $C_{\sigma}^{M}$ as follows:

Definition $3 \quad$ Let $\varepsilon \geq 0$ and $\sigma$ be a Cohen kernel such that $\int_{\mathbb{R}^{2 d}} \overline{\sigma(x, \omega)} d x d \omega=1$. We say that a function $f \in L^{2}\left(\mathbb{R}^{d}\right)$ is $\varepsilon$-concentrated on a measurable set $M \subseteq \mathbb{R}^{2 d}$ with respect to the concentration operator $C_{\sigma}^{M}$ if

$$
\left\|C_{\sigma}^{M} f\right\|_{2} \geq \sqrt{1-\varepsilon^{2}}\|f\|_{2}
$$

Theorem 3 Let $\sigma \in \mathcal{S}\left(\mathbb{R}^{d}\right) \backslash\{0\}$, and suppose that the corresponding Cohen representation $Q_{\sigma}$ is positive. Then

1) $C_{\sigma}^{M}$ is a trace class operator on $L^{2}\left(\mathbb{R}^{d}\right)$ and

$$
\left\|C_{\sigma}^{M}\right\|_{\operatorname{Tr}}=|M| \widehat{\sigma}(0,0)
$$

2) The following lower bound for the measure of the set M holds:

$$
|M| \geq \frac{1}{\widehat{\sigma}(0,0)} \sup _{\|f\|_{2}=1}\left\|C_{\sigma}^{M} f\right\|_{2} .
$$

3) If $f \in L^{2}\left(\mathbb{R}^{d}\right)$ is $\varepsilon$-concentrated on a $M \subseteq \mathbb{R}^{2 d}$ with respect to $C_{\sigma}^{M}$ (according to Definition 3) then

$$
|M| \geq \frac{1}{\widehat{\sigma}(0,0)} \sqrt{1-\varepsilon^{2}}
$$

Proof. If $Q_{\sigma}(f) \geqslant 0$ for all $f \in L^{2}\left(\mathbb{R}^{d}\right)$, then

$$
\left(C_{\sigma}^{M} f, f\right)=\iint_{M} \overline{Q_{\sigma}(f)(x, \omega)} d x d \omega \geqslant 0,
$$

so the concentration operator $C_{\sigma}^{M}$ is self-adjoint and positive. Therefore, writing, as well-known, the trace-class norm as integral on the diagonal of the kernel and using Proposition 7, we have

$$
\begin{aligned}
\left\|C_{\sigma}^{M}\right\|_{T r} & =\int_{\mathbb{R}^{d}} K_{M}(x, x) d x \\
& =\int_{\mathbb{R}^{d}} \mathcal{F}_{u \rightarrow x}\left[\check{\chi}_{M}(u, 0) \widehat{\sigma}(u, 0)\right] d x \\
& =\check{\chi}_{M}(0,0) \widehat{\sigma}(0,0) \\
& =|M| \widehat{\sigma}(0,0) .
\end{aligned}
$$

which proves (11).

The estimate (12) follows (11) and the fact that $\left\|C_{\sigma}^{M}\right\|_{T r} \geq\left\|C_{\sigma}^{M}\right\|_{B\left(L^{2}\right)}=\sup _{\|f\|_{2}=1}\left\|C_{\sigma}^{M} f\right\|_{2}$. Remark that if $\widehat{\sigma}(0,0)=$ 0 then necessarily $C_{\sigma}^{M}=0$ which is excluded in our hypothesis.

Finally the $\varepsilon$-concentration of a signal $f$ on a $M$ with respect to $C_{\sigma}^{M}$ implies $\sqrt{1-\varepsilon^{2}} \leq\left\|C_{\sigma}^{M}\right\|_{B\left(L^{2}\right)}$, from which estimate in (3) follows.

Remark $5 \quad$ Proposition 3 applies in particular to representations of the type $\left|Q_{\sigma}(f)\right|$, with $\sigma$ satisfying the hypothesis of Proposition 8.

\section{REFERENCES}

[1] M. Benedicks. On Fourier transforms of functions supported on sets of finite Lebesgue measure, J. Math. Anal. Appl. 106 (1), 180-183, 1985.

[2] P. Boggiatto, E. Carypis, and A. Oliaro. Windowed-Wigner representations in the Cohen class and uncertainty principles, J. Geom. Anal., 23 (4), 1753-1779, 2013. 
[3] P. Boggiatto, E. Carypis, and A. Oliaro. Local uncertainty principles for the Cohen class, J. Math. Anal. Appl, 419 (2), 1004-1022, 2014.

[4] P. Boggiatto, E. Carypis, and A. Oliaro. Two aspects of the Donoho-Stark uncertainty principle, J. Math. Anal. Appl, 434 (2), 1489-1503, 2016.

[5] P. Boggiatto, G. De Donno and A. Oliaro, Weyl quantization of Lebesgue spaces, Mathematische Nachrichten 282 (12), 1656-1663, 2009.

[6] P. Boggiatto, G. De Donno and A. Oliaro, Time-Frequency representations of Wigner type and Pseudodifferential Operators, Trans. Amer. Math. Soc. 362 (9), 4955-4981, 2010.

[7] P. Boggiatto, C. Fernández, A. Galbis. Supports of representations in the Cohen class, JFAA, 17 (6), 11801197, 2011.

[8] L. Cohen. Time-Frequency Analysis. Prentice Hall Signal Proc. series, New Jersey, 1995.

[9] L. Cohen. The uncertainty principle for the short-time Fourier transform Proc. Int. Soc. Opt. Eng., 22563, 80-90, 1995.

[10] M. A. de Gosson. Born-Jordan Quantization and the Uncertainty Principle. J. Phys. A: Math. Theor. 46, (2013)

[11] M. A. de Gosson. Introduction to Born-Jordan Quantization, Springer-Verlag, series Fundamental Theories of Physics (2015).

[12] D. L. Donoho, P. B. Stark. Uncertainty principles and signal recovery. SIAM J. Appl. Math., 49(3): 906-931, 1989.

[13] G. B. Folland, A. Sitaram. The uncertainty principle: a mathematical survey. J. Fourier Anal. Appl., 3(3), 207-238, 1989.

[14] K. Gröchenig. Foundations of Time-Frequency Analysis. Birkhäuser, Boston, 2001.

[15] A.J.E.M. Janssen. Bilinear phase-plane distribution functions and positivity. J. Math. Phys. 26 (8), 198619941985.

[16] A.J.A.M. Janssen. Proof of a conjecture on the supports of Wigner distributions. J. Fourier Anal. Appl., 4(6), 723-726, 1998.

[17] P. Korn. Some Uncertainty Principles for Time-Frequency Transforms of the Cohen Class. IEEE Transactions on Signal Processing , 53(2), 523-527, 2005.

[18] E.H. Lieb. Integral bounds for radar ambiguity functions and Wigner distributions. J. Math. Phys., 31(3), 594-599, 1990.

[19] M. A. Shubin. Pseudodifferential operators and spectral theory. Springer-Verlag, Berlin, second edition, 2001. Translated from the 1978 Russian original by Stig I. Andersson.

[20] M.-W. Wong Weyl Transforms. Universitext, Springer-Verlag, New York, 1998.

[21] M.-W. Wong Wavelet Transforms and Localization Operators. Birkhäuser, 2002.

[22] M.-W. Wong An introduction to pseudo-differential operators. Series on Analysis, Applications and Computation, 6. World Scientific Publishing Co. Pte. Ltd., Hackensack, NJ, 2014. 